\title{
Interacción social conflictiva y problemas de salud mental en redes personales de ex combatientes ilegales en Colombia
}

\section{Conflictive social interactions and mental health issues in personal networks of Colombian unlawful ex-combatants}

\author{
José Hernando Ávila-Toscano \\ Corporación Universitaria Reformada, Barranquilla, Colombia \\ Camilo Madariaga \\ Universidad del Norte, Barranquilla, Colombia
}

(Rec: 28 agosto 2015 / Acept: 25 octubre 2015)

\begin{abstract}
Resumen
Interacción conflictiva implica relaciones interpersonales en las que existen molestias o enojo. Este estudio abordó la existencia de diferencias significativas en la estructura de redes personales y problemas de salud mental de ex combatientes ilegales colombianos con y sin conflictos relacionales. Se evaluó a 106 individuos de tres ciudades mediante un estudio comparativo, analizando las propiedades estructurales de sus redes y el registro de problemas de sintomatología somática, ansiedad-insomnio, disfunción social y depresión grave. Las redes obtuvieron rendimiento moderado de sus indicadores, con mayor reporte de conflictos en aquellas con más cercanía y menor densidad relacional. Los problemas de ansiedad-insomnio y depresión fueron más comunes en redes con conflictos interpersonales. Las interacciones sociales mediadas por el conflicto parecen tener participación en los problemas de salud mental, por lo cual se sugiere incluir estrategias que fomenten interacciones cordiales dentro de las acciones tendientes a la adecuada reintegración de esta población.

Palabras clave: interacción social conflictiva, redes personales, salud mental y ex combatientes.
\end{abstract}

\begin{abstract}
A conflictive interaction implies interpersonal relationships where discomfort or anger feelings exist; this study addresses the existence of significant differences in the structure of personal nets and mental health problems in Colombian unlawful ex-combatants with and without relationships conflicts. 106 individuals were evaluated in three cities throughout a comparative study, analyzing the structural properties of their personal networks and the registration of problems as somatic symptomatology, anxiety-insomnia, social dysfunction and severe depression. The networks shown moderate performance of their indicators, reporting higher number of conflicts in those where the closeness is higher and the relational density is lower. Anxiety-Insomnia and depression problems were more common in networks with interpersonal conflicts. Findings suggest that social interactions mediated by the conflict seem to have participation in mental health problems, reason why it is suggested to include strategies that encourage positive interactions related to the proper reintegration of this population.

Keywords: conflictive social interactions, personal networks, mental health, ex-combatants.
\end{abstract}

\footnotetext{
Este artículo se desarrolló en el marco del Doctorado en Ciencias Humanas y Sociales de la Universidad Nacional de Misiones (Posadas, Argentina). Correspondencia: Carrera 38 № 74-179, Barranquilla, Colombia. javila@unireformada.edu.co
} 


\section{Introducción}

Los reportes acerca de la manera como las propiedades de las redes influyen favorable o desfavorablemente en la salud psicoemocional son frecuentes. En su mayoría, estos estudios reconocen que contar con redes amplias, con intercambios funcionales y con buenos niveles de apoyo social, contribuye con la felicidad y el capital social, la salud física y mental, aumenta el bienestar, ayuda a afrontar el estrés, reduce la morbimortalidad y aumenta la satisfacción vital (Iwasaki et al., 2002; Brissette, Scheier y Carver, 2002; Gallicchio, Hoffman y Helzlsouer, 2007, Lahuerta, Borrell, Rodríguez-Sanz, Pérez y Nebot, 2004; Hernández, Pozo, Morillejo y Martos, 2005; Cohen y Janicki-Deverts, 2009; Thoits, 2011; Litwin y Shiovitz-Ezra, 2011; Vivaldi y Barra, 2012; San Martín y Barra, 2013), mientras que carecer de fuentes de apoyo producto de tener redes pequeñas o deficitarias disminuye la salud física percibida, la salud mental y la vitalidad, a la vez que aumenta la desesperanza y la depresión (Achat et al., 1998; Cornwell y Waite, 2009; Golden et al., 2009).

De este modo, la mayor parte de los estudios se concentran en el efecto positivo o negativo de contar o no con una estructura social en la que existan mecanismos cotidianos de apoyo (Matud, Caballeira, López, Marrero e Ibáñez, 2005), pero un indicador menos registrado en la literatura especializada consiste en cómo las propiedades estructurales de las redes pueden tener influencia en el desarrollo de pautas relacionales conflictivas independientemente de que se trate de redes de menor o mayor tamaño, así como la interacción de dichas relaciones conflictivas con la salud mental de los individuos.

Los vínculos conflictivos se refieren a aquellas relaciones en las que surgen molestias o enojo, que pueden darse incluso con los mismos proveedores de apoyo (Barrera, 1980). Desde finales de la década de 1970 se reconocen reportes empíricos que se centraban en el análisis de las interacciones sociales desagradables. Henderson et al. (1978), por ejemplo, identificaron que aquellos individuos que experimentaban más conflictos en sus relaciones mostraban un mayor nivel de morbilidad psiquiátrica. De este modo, las molestias interpersonales se reconocieron como una variable capaz de tener efectos nocivos sobre el bienestar.

En una red social es esperable el surgimiento de interacciones negativas en función de los lazos de intimidad que se entretejen. García et al. (2002) han expresado que el surgimiento de tensiones suele ser constante en las redes con alta integración de familiares, lo cual se puede asociar con las expectativas de éxito del proyecto familiar. A su vez, Fisher, Reid y Meléndez (1989) identificaron que los conflictos también son frecuentes con las amistades y señalaron que las causas principales incluyen irritación al sentir que los amigos no están a la altura de las expectativas del rol, envidia en temas financieros, etc. Otras fuentes han demostrado que las relaciones conflictivas ejercen un fuerte efecto negativo sobre la percepción de la calidad de vida, e incluso afectan el nivel de satisfacción vital y reducen la autoestima (Rosenfield y Wenzel, 1997), Asimismo, las tensiones relacionales se han asociado con un mayor riesgo de problemas de salud mental tanto en hombres como en mujeres (Stansfeld, Fuhrer y Shipley, 1998).

En este estudio, el foco de análisis acerca de las interacciones sociales conflictivas (en adelante ISC) se centra en las redes personales de ex combatientes ilegales en Colombia, al igual que en la identificación de problemas en su salud mental. Las evidencias empíricas con ex combatientes colombianos son diversas. En materia de sus redes se ha reportado su tendencia a conformar estructuras pequeñas, con baja densidad relacional y preferencia de integración con familiares y amigos cercanos, además, estructuralmente muestran niveles medio-bajos en sus indicadores de centralidad (Amar-Amar, Abello-Llanos, Madariaga y Ávila-Toscano, 2011; Álvarez y Guzmán, 2013; Amar-Amar, Abello-Llanos, Madariaga y Ávila-Toscano, 2014). En gran medida, su estrechez y bajo tamaño podrían estar relacionados con la falta de confianza y el rechazo del que son objeto por parte del medio social (Nussio, 2011).

En relación con su salud mental, fuentes internacionales señalan como frecuentes los problemas relacionados con el consumo de drogas, la depresión y el suicidio (Nilsson, 2005; Winkler, 2010), estrés postraumático y sentimientos de venganza (Bayer, Klasen y Adam, 2007). Los estudios con ex combatientes colombianos señalan problemas similares: estrés postraumático, consumo de sustancias psicoactivas, retardo mental, trastornos psicóticos (De la Espriella y Falla, 2009; Aristizábal et al., 2012), entre otros.

El estudio de los conflictos relacionales frente a la estructura de las redes y la salud mental de ex combatientes no cuenta con registros científicos en Colombia, precisamente por ello, en este estudio el objetivo planteado consiste en establecer si existen diferencias significativas en la estructura de las redes personales y en las problemáticas de salud mental de ex combatientes, de acuerdo con la presencia o ausencia de ISC en dichas redes. 


\section{Método}

\section{Participantes}

A través de una investigación empírica de diseño comparativo (Ato, López y Benavente, 2013) se evaluó a 106 ex combatientes de grupos ilegales colombianos debidamente desmovilizados e incluidos en el proceso de reintegración a la vida civil. Los participantes fueron seleccionados en tres ciudades del caribe colombiano, 34 de Barraquilla, 38 de Santa Marta y 34 de Montería; $92.5 \%(n=98)$ eran hombres y $7.5 \%(n=8)$ mujeres, con una media de edad de 35.8 años $(d e=6.9)$ y una media de tiempo de desmovilización de 8.4 años $(d e=1.7)$.

La selección se hizo entre las personas activas en el proceso de reintegración de cada ciudad, sin diferenciación de su nivel de avance en dicho proceso, con individuos que no registraban problemas de seguridad, ubicados en las zonas urbanas y con un tiempo mínimo de residencia de seis meses en las mismas.

\section{Instrumentos}

La estructura de las redes personales y el registro de ISC se cumplió con el Arizona Social Support Interview Schedule (ASSIS) (Barrera, 1980), diseñado para registrar la red de contactos y los mecanismos de apoyo social de las mismas, así como el reporte de conflictos entre los integrantes, tanto de los conflictos esperados como de los realmente experimentados en el mes previo a la evaluación. La evidencia empírica demuestra un rendimiento positivo a nivel psicométrico del ASSIS (confiabilidad test - re test de .88 luego de tres días y de.70 pasado un mes) (López et al., 2007), con amplio uso y aplicación en población colombiana (Amar-Amar et al., 2011 y 2014; Álvarez y Guzmán, 2013).

Las problemáticas relacionadas con la salud mental se evaluaron a través del Cuestionario de Salud General en su versión de 28 ítems (General Health Questionnaire, GHQ28 ), pues cuenta con validez transcultural siendo uno de los instrumentos más empleados en Colombia para medir la salud mental (Gómez-Ortiz y Moreno, 2009; Juárez y Guerra, 2011). Identifica cuatro dimensiones problemáticas, denominadas sintomas somáticos, ansiedad-insomnio, disfunción social y depresión grave, a través de una escala tipo Likert de 4 opciones.

\section{Procedimiento}

Para su ejecución, el estudio contó con la aprobación oficial de la Agencia Colombiana para la Reintegración (ACR), lo que permitió el acceso a los centros de atención de ex combatientes en cada ciudad. El estudio se ajustó a un protocolo ético acorde con lo dispuesto en la Ley 1090 de 2006, emanada del Congreso de la República de Colombia, por la cual se reglamenta el ejercicio de la profesión de psicología en este país, se dicta el Código Deontológico y Bioético y otras disposiciones.

Los participantes se vincularon de forma voluntaria y autónoma, con pleno reconocimiento de los objetivos y fines del estudio y con conciencia de que su cooperación no implicaba beneficios económicos. La evaluación se cumplió en los centros de atención de la ACR, garantizando la privacidad y reserva de la información obtenida. El levantamiento de la información fue realizado por un grupo de cinco psicólogos con experticia en el uso de los instrumentos y experiencia en el trabajo con esta población.

Los datos relacionados con las redes personales se analizaron con el software Ucinet versión 6.391 (Borgatti, Everett y Freeman, 2002) para calcular los indicadores estructurales (grado nodal, cercanía, intermediación, clúster, densidad y centralización), que permiten conocer la forma en que las mismas se organizan, los procesos de interacción y la conformación de relaciones. Asimismo, la información acerca de su composición, la ISC y la salud mental se analizó descriptivamente con SPSS 19.0. Posteriormente se aplicó la prueba de contraste entre dos grupos independientes (U de Mann-Whitney) para identificar si los indicadores estructurales y los problemas de salud mental mostraban diferencias significativas entre las redes que expresaban conflicto y las que no.

\section{Resultados}

Según su composición, $56 \%$ de los integrantes de las redes analizadas fueron hombres y $44 \%$ mujeres, sobresaliendo la preferencia por personas allegadas, especialmente por individuos a los que se conoce desde hace varios años, así, las redes estuvieron integradas en un $48.4 \%(n=1019)$ por amigos muy cercanos, $40.3 \%(n=854)$ por familiares, $7.5 \%$ $(n=159)$ por conocidos y $2.6 \%(n=55)$ de los miembros eran las parejas de los ex combatientes. Adicionalmente, el 
$1.4 \%(n=30)$ de los integrantes de estas redes correspondió a funcionarios del programa de reintegración considerados como contactos proveedores de apoyo.

En cuanto a lo estructural, el resumen de los resultados descriptivos por cada indicador de las redes se encuentra en la Tabla 1. Esta información denota que las redes se caracterizaron por poseer un nivel moderado de rendimiento en sus indicadores de centralidad. En tanto, los resultados obtenidos en el grado nodal, la centralización y la densidad sugieren que se trata de redes en las que existe un grupo determinado de personas que concentran gran porción de las relaciones, con popularidad por parte de los actores principales, es decir, los ex combatientes, quienes parecen gozar de un margen de interacciones importante.

En estas redes se aprecia la tendencia a conformar subagrupaciones especialmente en relación con la similitud compartida con ciertos actores (según los valores del coeficiente de clúster) y a su vez el rendimiento de la intermediación señala que, así como existe apertura hacia la integración de nuevos actores en algunas redes, otras son un tanto más restringidas, aunque en términos generales parece existir un potencial de conformación de interacciones con sujetos nuevos, esto es, no se trata de redes completamente cerradas. Adicionalmente, el nivel de cercanía indica que la integración se organiza de manera diversa de acuerdo con la distancia social de los contactos, lo que quiere decir que hay un buen número de contactos poco cercanos, lo cual coincide con la descripción previa de ser redes con escasa tendencia al cierre, esto en la medida que las relaciones pueden sostenerse en un marco de distanciamiento psicosocial.

Por su parte, la evaluación de existencia de formas de ISC permitió identificar diferencias entre las discusiones, riñas y demás dificultades personales esperadas y las realmente vividas en relación con el mes previo a la evaluación. Se observó que en $73.6 \%(n=78)$ de las redes se esperaba tener interacción negativa, mientras que en el último mes

Tabla 1. Resumen de los estadísticos descriptivos de los indicadores estructurales evaluados en las redes personales de ex combatientes.

\begin{tabular}{lcccc}
\hline Indicador & Mínimo & Máximo & Media & DE \\
\hline Grado nodal & .00 & 20.00 & 13.1 & 5.6 \\
Clúster & .00 & 1.00 & .8 & .1 \\
Densidad & .00 & 1.00 & .6 & .2 \\
Centralización & .00 & 75.90 & 20.1 & 19.2 \\
Cercanía & .00 & 298.20 & 50.4 & 64.5 \\
Intermediación & .00 & 29.70 & 3.6 & 4.8 \\
\hline
\end{tabular}

Fuente: elaboración propia.

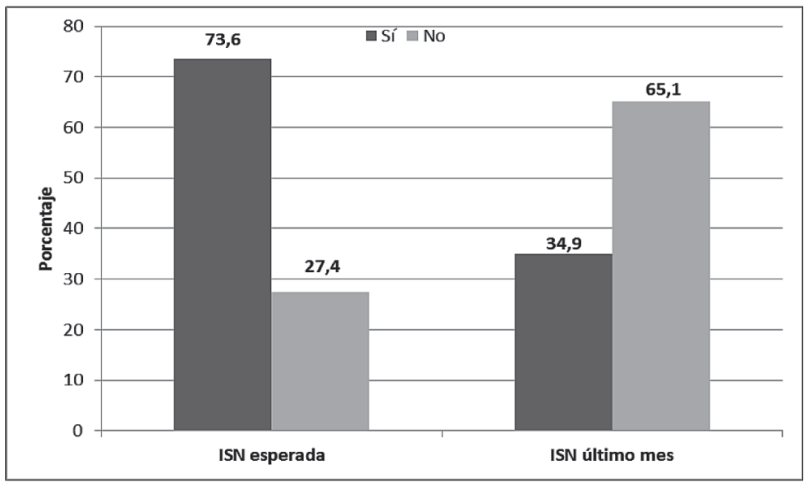

Figura 1. Comparación entre la Interacción Social Conflictiva (ISC) esperada y experimentada el último mes previo a la evaluación. Fuente: elaboración propia.

el reporte de problemáticas en las relaciones fue del 34.9\% $(n=37)$. Esta información aparece en la Figura 1.

En la valoración de problemáticas relacionadas con la salud mental, la disfunción social fue la condición más frecuente $(\bar{X}=5.6, d e=2.0)$ al ser reportada en $41.5 \%(n=44)$ de los participantes, mientras que los Sintomas somáticos obtuvieron una media de $4.48(d e=1.8)$ y se reportaron en $15.1 \%(n=16)$ de los evaluados. La subescala de AnsiedadInsomnio obtuvo una media de $3.0(d e=1.8)$ y un porcentaje de aparición de $5.7 \%(n=6)$ y finalmente, la media más baja de los cuatro indicadores fue la de Depresión grave con un valor de $1.5(d e=1.4)$ y sólo se reportó en $1.9 \%(n=2)$ de los ex combatientes.

Por otro lado, en el análisis sobre diferencias significativas entre las variables estudiadas en relación con la ISC, se procedió a contrastar el reporte de conflictos reales, es decir, los ocurridos en el último mes con los indicadores estructurales de red y su composición. Frente a esta última variable no se hallaron datos estadísticamente relevantes de tal forma que la ISC no mostró diferencias significativas en redes con más o menos familiares $(U=1172.0, Z=-.694 \mathrm{p}>$ $.05)$ o amistades $(U=1068.5, Z=-1.383 \mathrm{p}>.05)$. Los resultados para las demás variables se encuentran en la Tabla 2.

El análisis de contraste de medias identificó tres diferencias significativas. Inicialmente se observa un menor nivel de rendimiento del grado nodal ( $\mathrm{rm}=44.45)$ y más baja densidad $(r m=44.38)$ en las redes en las que existe reporte de ISC, mientras que en las estructuras sin este tipo de interacción el rendimiento de tales indicadores es mayor $(r m=58.36 \mathrm{y}$ $r m=58.69$ respectivamente). Como contraparte, la cercanía muestra valores significativamente más altos en el primer grupo de redes $(r m=62.31)$ que en el segundo $(r m=48.78)$. 
Tabla 2. Prueba de contraste de medias entre interacción social negativa e indicadores estructurales de redes.

\begin{tabular}{lcc}
\hline \multirow{2}{*}{ Variables de contraste } & \multicolumn{2}{c}{ Estadísticos de contraste } \\
\cline { 2 - 3 } & U Mann-Whitney & $Z$ \\
\hline Grado nodal & $941.50^{*}$ & -2.232 \\
Clúster & 993.5 & -1.891 \\
Densidad & $939.0^{*}$ & -2.25 \\
Centralización & 1079.5 & -1.314 \\
Cercanía & $950.50^{*}$ & -2.173 \\
Intermediación & 1032.5 & -1.632 \\
\hline
\end{tabular}

Variable de agrupación: Interacción social conflictiva.

Nota: ${ }^{*} \mathrm{p}<.05$. Fuente: elaboración propia.

Por último, este mismo procedimiento halló dos diferencias significativas al contrastar la ISC con los problemas de salud mental. La información obtenida señala que las manifestaciones de Ansiedad-Insomnio son mayores en redes con reporte de conflictos $(r m=61.85)$ que en redes sin interacción conflictiva $(r m=49.02)(U=967.50, Z=-$ $2.090 \mathrm{p}<.05)$, e idéntica situación ocurrió con los niveles de depresión grave $(U=1062.50, Z=-2.039 \mathrm{p}<.05)$.

\section{Discusión}

El desarrollo de este estudio se basó en la identificación de diferencias significativas en la estructura de las redes personales y la manifestación de problemas de salud mental en ex combatientes ilegales, en función de la presencia o ausencia de conflictos en las relaciones sostenidas con los integrantes de dichas redes. La evidencia obtenida por un lado respalda hallazgos previos en ex combatientes colombianos en los que se resaltan propiedades estructurales con un nivel de rendimiento moderado (Álvarez y Guzmán, 2013), redes con alta concentración de personas con las cuales hay un vínculo estrecho generalmente mediado por el lazo filial o la cercanía emocional que data de varios años de amistad (Amar-Amar et al., 2011 y 2014), si bien en nuestro caso, las redes identificadas expresaron un desempeño más favorable a nivel de sus elementos estructurales y la conformación muestra una mayor integración con amistades que con la propia familia.

Una factible explicación radica en que los estudios previos se han ejecutado principalmente en zonas urbanas de pequeñas municipalidades colombianas tomadas como zonas de reasentamiento paramilitar principalmente, mientras que el actual estudio se cumplió en ciudades medianas y grandes, lo cual implica un aumento de la distancia geográfica con los contactos y especialmente variabilidad en la procedencia dado que, en los estudios de Amar-Amar et al. (2011 y 2014), la población se basó en ex combatientes que retornaban a sus núcleos sociales de origen, mientras que en las ciudades abordadas en nuestro caso es común hallar desmovilizados procedentes de múltiples zonas de Colombia. Previamente, Álvarez y Guzmán (2013) han supuesto una postura similar, al observar diferencias en las redes de desmovilizados de zonas periféricas frente a los que viven en ciudades grandes.

Sin embargo, es innegable la cercanía de los resultados en los diversos estudios frente a la estructura general de las redes, aunque en nuestro caso el enfoque no se haya centrado en los medios de apoyo social sino en la existencia de relaciones mediadas por el conflicto. Al respecto, resulta llamativo identificar una mayor tendencia a esperar este tipo de situaciones que a experimentarlas, de tal suerte que entre los ex combatientes se percibe un porcentaje alto (73.6\%) de potenciales contactos conflictivos. Algunos autores han señalado que la integración social (y comunitaria) no es uno de los procesos más fuertes en desmovilizados (Gleichmann, Odenwald, Steenken y Wilkinson, 2004), siendo comunes los conflictos con la comunidad o la baja participación dentro de la misma (Kaplan y Nussio, 2012). Asimismo, se ha señalado que las interacciones negativas en esta población son esperables con familiares pero sustancialmente con la pareja, aunque esto es un indicador de relativa normalidad dentro de las relaciones conyugales (Ávila-Toscano y Madariaga, 2010). Sin embargo, la integración de más familiares o más amigos en las redes no resulta ser un elemento significativo para suponer que generan diferencias en cuanto a la aparición de conflictos, por lo cual los mismos parecen darse en estas redes independientemente de la forma como estén compuestas, lo cual constituye un hallazgo que difiere con lo descrito por García et al. (2002) y por Fisher et al. (1989), para quienes la participación de más familiares o más amistades respectivamente contribuye al surgimiento de interacciones negativas o desagradables.

La composición de las redes, por tanto, no cumple un papel relevante en la presencia o no de conflictos, en cambio las propiedades estructurales sí desempeñan un rol esencial. En este estudio, las redes con mayor reporte de relaciones conflictivas son aquellas en las que la densidad relacional es menor, al igual que la centralidad relativa a cada actor, en cambio, son redes con altos niveles de cercanía, lo que indica que en las redes con más estrechamiento en las relaciones donde los lazos principales se encuentran definidos en pocos contactos y no existe apertura social es más común hallar muestras de tensión, lo cual constituye una evidencia adicional a la desventaja que representa contar con redes estrechas o encapsuladas (Cornwell y Waite, 2009; Golden et al., 2009). Para el caso de los ex combatientes, esta 
información supone la necesidad de estimular el flujo de relaciones en escenarios sociocomunitarios más amplios, que no se restrinjan exclusivamente a los lazos conformados con los allegados sino que impliquen la posibilidad de darse participación pública a través del contacto con nuevos miembros de las comunidades, condiciones que influyen en la posibilidad de ganar integración comunitaria (Nilsson, 2005; Kaplan y Nussio, 2012) indispensable en la aspiración de reintegrarse.

Las redes con y sin interacciones conflictivas también mostraron diferencias en lo referente a la salud mental de los ex combatientes. En un principio es necesario indicar que las problemáticas identificadas no tuvieron una frecuencia de aparición elevada. Curiosamente, fue la disfunción social la más reseñada, pero condiciones como la depresión grave y la ansiedad e insomnio no tuvieron una alta tasa de reporte, lo cual difiere con estudios anteriores (Winkler, 2010; Bayer et al., 2007). Sin embargo, también se ha descrito que entre esta población es común hallar una respuesta reacia a manifestar sus problemas sintomáticos (De la Espriella y Falla, 2009); asumirlos es visto como una señal de debilidad dentro de un conglomerado social en el que priman imaginarios de comportamiento que priorizan la masculinidad y la fuerza (De la Espriella y Falla, 2009), ello conduce a que el bajo reconocimiento de los problemas psicoemocionales reduzca la posibilidad de registro de los mismos.

La información relacionada con la existencia de interacciones conflictivas y los problemas en la salud mental refuerzan los resultados reportados por estudios anteriores (Rosenfield y Wenzel, 1997; Stansfeld, 1998) en la medida que en las redes con este tipo de interacción se identifica una mayor frecuencia de problemas de ansiedad e insomnio y de síntomas depresivos, lo cual parece sugerir que la existencia de relaciones mediadas por la tensión o los problemas interpersonales pueden contribuir con el surgimiento de inestabilidad en las respuestas psicológicas de los individuos. En este sentido, los recursos sociales de apoyo con los cuales cuentan las personas deben cumplir un rol amortiguador capaz de reducir el impacto nocivo de los conflictos relacionales.

No obstante, una visión crítica de los procedimientos cumplidos conduce a reconocer limitaciones frente a los hallazgos obtenidos. La naturaleza transversal de este estudio no deja reconocer los posibles cambios en el patrón de reporte de conflictos efectivamente registrados, de esta manera no es posible definir si la ISC reportada en el mes previo responde a un patrón que se sostiene en el tiempo o si obedece a condiciones propias del contexto relacional ocurridas en el período evaluado. Del mismo modo, nuestros reportes se basan en definir si existieron o no tales conflictos, pero no se realizó una precisión de cuáles fueron o los motivos asociados, lo que podría ofrecer una mayor riqueza en la comprensión de los elementos que intervienen en las tensiones relacionales de los ex combatientes.

A pesar de lo anterior, la información aquí presentada constituye una nueva evidencia acerca del proceso de socialización y construcción de estructuras sociales en personas que han dejado el ejercicio ilegal de las armas y que se encuentran dentro de un proceso de reintegración. Precisamente, frente a dicho proceso se pueden asumir los principales aportes de estudios como el presentado, en la medida que el reconocimiento de conflictos potenciales y reales y la diferenciación de su participación en el desarrollo o no de problemas de salud mental, constituye una contribución valiosa para la formulación de estrategias de fomento de pautas relacionales basadas en la resolución no violenta de conflictos, el uso de mecanismos de negociación ante las tensiones interpersonales y demás herramientas que puedan coadyuvar a sostener relaciones sociales más amenas, cordiales y libres de disputas, esperando con ello una intervención indirecta sobre el desempeño de la salud psicológica de quienes aspiran reintegrarse.

\section{Referencias}

Achat, H., Kawachi, I., Levine, S., Berkey, C., Coakley, E. y Colditz, G. (1998). Social Networks, Stress and Health-Related Quality of Life. Quality of Life Research, 7, 735-750.

Álvarez, E. y Guzmán, G. (2013). Redes de apoyo social en personas en proceso de reintegración a la vida civil residentes en la ciudad de Barranquilla (Colombia). Realitas, Revista de Ciencias Sociales, Humanas y Artes, 1, 11-17.

Amar-Amar, J., Abello-Llanos, R., Madariaga, C. y Ávila-Toscano, J. H. (2011). Relación entre redes personales y calidad de vida en desmovilizados del conflicto armado colombiano. Universitas Psychologicas, $10,355-369$.

Amar-Amar, J., Abello-Llanos, R., Madariaga, C. y Ávila-Toscano, J. H. (2014). Characteristics of personal networks associated with physical and psychological quality of life among demobilized individuals from the Colombian armed conflict. Journal of Community y Applied Social Psychology, 24, 173-190. doi: 10.1002/casp.2155.

Aristizábal, E., Palacio, J., Madariaga, C., Osman, H., Parra, L. Rodríguez, J. y López, G. (2012). Síntomas y traumatismo psíquico en víctimas y victimarios del conflicto armado en el Caribe colombiano. Psicología desde el Caribe, 29, 123-151.

Ato, M., López, J. J. y Benavente, A. (2013). Un sistema de clasificación de los diseños de investigación en psicología. Anales de psicología, 29, 1038-1059.

Ávila-Toscano, J. H. y Madariaga, C. (2010). Redes personales y dimensiones de apoyo en individuos desmovilizados del conflicto armado. Psicología desde el Caribe, 25, 179-201.

Barrera, M. (1980). A method for the assessment of social support networks in community survey research. Connections, 3, 8-13.

Bayer, C. P., Klasen, F. y Adam, H. (2007). Association of trauma and PTSD symptoms with openness to reconciliation and feelings of revenge 
among former Ugandan and Congolese child soldiers. Journal of the American Medical Association, 298, 555-559.

Borgatti, S. P., Everett, M. G. y Freeman, L. C. (2002). Ucinet for Windows: Software for Social Network Analysis. Harvard, MA: Analytic Technologies.

Brissette, I., Scheier, M. F. y Carver, C. S. (2002). The role of optimism in social network development, coping, and psychological adjustment during a life transition. Journal of Personality and Social Psychology, $82,102-111$.

Cohen, S. y Janicki-Deverts, D. (2009). Can we improve our physical health by altering our social networks? Perspectives on Psychological Science, 4, 375-378. doi: 10.1111/j.1745-6924.2009.01141.x.

Cornwell, E. Y. y Waite, L. (2009). Social disconnectedness, perceived isolation, and health among older adults. Journal of Health and Social Behavior, 50, 31-48. doi: 10.1177/002214650905000103.

De la Espriella, R. y Falla, J. (2009). Reflexiones sobre la atención en salud mental de desmovilizados de grupos armados en Colombia. Revista Colombiana de Psiquiatría, 38, 230- 247.

Fisher, C. B., Reid, J. D. y Meléndez, M. (1989). Conflict in families and friendships in later life. Family Relations, 38, 83-89.

Gallicchio, L., Hoffman, S. y Helzlsouer, K. (2007). The relationship between gender, social support, and health- related quality of life in a community-based study in Washington County, Maryland. Quality of life research, 16, 777-786.

García, M., Martínez, M. y Albar, M. (2002). La elección de fuentes de apoyo social entre inmigrantes. Psicothema, 14, 369-374.

Gleichmann, C., Odenwald, M., Steenken, J. y Wilkinson, A. (2004). Disarmament, Demobilisation and Reintegration. A practical field and classroom guide. Frankfurt, Alemania: Druckerei Hassmüller Graphische Betriebe $\mathrm{GmbH}$ y Co. KG.

Golden, J., Conroy, R., Bruce, I., Denihan, A., Greene, E., Kirby, M. y Lawlor, B. (2009). Loneliness, social support networks, mood and wellbeing in community-dwelling elderly. International Journal of Geriatric Psychiatry, 24, 694-700.

Gómez-Ortiz, V. y Moreno, L. (2009). Factores psicosociales del trabajo (demanda-control y desbalance esfuerzo-recompensa), salud mental y tensión arterial: un estudio con maestros escolares en Bogotá, Colombia. Universitas Psychologica, 9, 393-407.

Henderson, S., Byrne, D.G., Duncan-Jones, P., Adcock, S., Scott, R. y Steele, G.P. (1978). Social Bonds in the Epidemiology of Neurosis: A Preliminary Communication. British Journal of Psychiatry, 132, 463-466.

Hernández, S., Pozo, S., Morillejo, E. y Martos, M. J. (2005). Estructura y funciones del apoyo social en un colectivo de inmigrantes marroquíes. Anales de Psicología, 21, 304-315.

Iwasaki, M., Otani, T., Sunaga, R., Miyazaki, H., Xiao, L., Wang, N., Yosiaki, S. y Suzuki, S. (2002). Social networks and mortality based on the Komo-Ise cohort study in Japan. International journal of epidemiology, 31, 1208-1218.

Juárez, F. y Guerra, Á. (2011). Características socioeconómicas y salud en personas pobres y desplazadas. Psicologia: Teoria e Pesquisa, 27, 511-519.

Kaplan, O. y Nussio, E. (2012). Community Counts: The Social Reintegration of Ex-combatants in Colombia. Annual Meeting of the American Political Science Association. New Orleans, USA.

Lahuerta, C., Borrell, C., Rodríguez-Sanz, M., Pérez, K. y Nebot, M. (2004). La influencia de la red social en la salud mental de la población anciana. Gaceta sanitaria, 18, 83-91.

Litwin, H. y Shiovitz-Ezra, S. (2011). Social network type and subjective well-being in a national sample of older Americans. The Gerontologist, 51, 379-388. doi: 10.1093/geront/gnq094.

López, I., Álvarez, S., Lorence, B., Jiménez, L., Hidalgo, M. y Sánchez, J. (2007). Evaluación del apoyo social mediante la escala ASSIS: descripción y resultados en una muestra de madres en situación de riesgo psicosocial. Intervención Psicosocial, 16, 323-337.

Matud, P., Caballeira, M., López, M., Marrero, S. e Ibáñez, I. (2005). Salud Mental, 25, 35-37.
Nilsson, A. (2005). Reintegrating Ex-Combatants in Post-Conflict Societies. Department for Cooperation with Non-Governmental Organisations and Humanitarian Assistance \& Conflict Management. Swedish: Edita communication.

Nussio, E. (2011). Understanding Ex-combatants. Central themes on the lives of paramilitaries of Colombia (Tesis de doctorado). University of St. Gallen. Switzerland.

Rosenfield, S. y Wenzel, S. (1997). Social Networks and Chronic Mental Illness: A Test of Four Perspectives. Social Problems, 44, 200-216.

San Martín, J. L. y Barra, E. (2013). Autoestima, apoyo social y satisfacción vital en adolescentes. Terapia psicológica, 31, 287-291. http://dx.doi. org/10.4067/S0718-48082013000300003.

Stansfeld, S. A., Fuhrer, R. y Shipley, M. J. (1998). Types of social support as predictors of psychiatric morbidity in a cohort of British Civil Servants (Whitehall II Study). Psychological Medicine, 28, 881-892.

Thoits, P. (2011). Mechanisms linking social ties and support to physical and mental health. Journal of Health and Social Behavior, 52, 145-161.

Vivaldi, F. y Barra, E. (2012). Bienestar psicológico, apoyo social percibido y percepción de salud en adultos mayores. Terapia psicológica, 30, 23 29. http://dx.doi.org/10.4067/S0718-48082012000200002.

Winkler, N. (2010). Psycho-social intervention needs among ex-combatants in Southern Sudan. Research Report. Juba: Southern Sudan DDR Commission (SSDDRC), Bonn International Center for Conversion (BICC). 


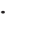

